\title{
*RADIO PULSARS IN THE MAGELLANIC CLOUDS
}

\author{
D. MCCONNELL 1 , P.M. MCCULLOCH ${ }^{2}$, P.A. HAMILTON ${ }^{2}$, J.G. ABLES ${ }^{3}$, \\ P.J. HALL 1 , C.E. JACKA ${ }^{3}$, A.J. HUNT 4 \\ $\dagger 1$ Australia Telescope National Facility, CSIRO, PO Box 94, Narrabri, NSW 2390, Australia \\ 2Department of Physics, University of Tasmania, PO Box 252C, Hobart, Tas., Australia \\ 3Division of Radiophysics, CSIRO, PO Box 76, Epping, NSW 2121, Australia \\ $\nmid 4$ Australia Telescope National Facility, CSIRO, PO Box 276, Parkes, NSW 2870, Australia
}

\begin{abstract}
The Large Magellanic Cloud (LMC) and Small Magellanic Cloud (SMC) have been searched for radio pulsars using the 64-m Parkes radiotelescope. The search has resulted in the discovery of four pulsars. Observed dispersion measures to each suggest that three lie in the Clouds (two in the LMC, one in the SMC) and that the fourth, which was found in the direction of the LMC, is a foreground object belonging to the galactic pulsar population.
\end{abstract}

*Paper submitted to M.N.R.A.S.

†The Australia Telescope National Facility is operated in association with the Division of Radiophysics by CSIRO. 